\title{
Neuronal Activity in Human Lateral Temporal Cortex during Serial Retrieval from Short-Term Memory
}

\author{
Michael M. Haglund, George A. Ojemann, Theodore W. Schwartz, and Ettore Lettich \\ Department of Neurological Surgery, University of Washington, Seattle, Washington 98195
}

Neuronal activity was recorded extracellularly from 20 populations in the lateral cortex of the left anterior temporal lobe of 11 patients undergoing awake craniotomy for epilepsy, during an input-distraction-retrieval measure of recent verbal memory that also included two later successive retrievals of the same information after additional distracting tasks. Changes in activity were determined for each 1 sec epoch in three major comparisons: (1) the same visual cues used for naming an input to recent memory, naming without a memory component, and a spatial matching task; (2) memory input (MI), distraction (S), and initial cued retrieval (R1) from memory, where object naming was the input to memory and naming of other objects the distractors; (3) initial retrieval (R1) and the two subsequent serial retrievals of the same information (R2, R3). Control comparisons were also made with serial naming and viewing of blank slides, and repeated naming of the same objects. In comparison 1, 13 of the 20 populations showed consistently increased activity during memory input ("'memory units"); two others showed changes during language measures. In comparison 2, a significant proportion of all 20 populations, and the 13 memory units considered alone showed increased activity in initial epochs of MI and R1, confirming earlier findings of increased lateral temporal neuronal activity at memory entry and initial retrieval. In comparison 3, a significant proportion of the memory units showed increased activity in early epochs of R1 and decreased activity in late epochs of R3. This decrease in populations with increased activity at R1 was also evident when $R 1$ was compared to $R 2$ or $R 2$ to $R 3$. Control comparisons showed no evidence of general habituation or decline in activity. Increased neuronal activity occurs in many left temporal neocortical neurons with input and the initial retrieval of an item from recent verbal memory, and the activity fades rapidly with repeated retrievals.

IKey words: human, temporal cortex, temporal lobe, recent verbal memory, short-term memory, microelectrode, singleunit recordings, awake craniotomy]

An important role for the temporal lobe in recent memory is generally accepted (Milner, 1971). This role has been further defined in man as one for memory for explicit material that

Received Feb. 2, 1993; revised July 13, 1993; accepted Aug. 26, 1993.

This work was supported by NIH Grant NS21724 and the American Association of Neurological Surgeons Research Foundation. We thank Doug Kalk for computer support/programming and Susan Perkins for secretarial assistance.

Correspondence should be addressed to George A. Ojemann, M.D., Professor of Neurological Surgery, Department of Neurological Surgery, RI-20, University of Washington, Seattle, WA 98195.

Copyright (C) 1994 Society for Neuroscience $0270-6474 / 94 / 141507-09 \$ 05.00 / 0$ must be retained during a distraction produced by similar material (Squire, 1987), with this type of memory for verbal material related to the left temporal lobe and that for visuospatial material to the right (Milner, 1971). Usually the medial structures of the temporal lobe are assigned this role, especially the hippocampus. However, there is also evidence for lateral temporal neocortex involvement in recent memory derived from effect of lesions in primates (Fuster et al., 1981) and humans (Milner, 1967; Ojemann and Dodrill, 1987), stimulation mapping in humans (Fedio and Van Buren, 1974; Ojemann, 1978, 1983; Ojemann and Dodrill, 1985), and recording of changes in activity of neurons in lateral temporal cortex during memory measures in primates (Fuster and Jervey, 1982) and humans (Ojemann et al., 1988, 1990).

The one study of neuronal activity in lateral neocortex of human left temporal lobe during a recent verbal memory measure frequently recorded increased activity when information entered memory and when it was retrieved but not throughout the period during which it was stored (Ojemann et al., 1988). That study investigated changes in neuronal activity only during a single initial retrieval of an item stored in recent verbal memory. The present study was directed at two issues related to this left temporal neocortical role in recent verbal memory: first, replication of the changes in neuronal activity in another series of patients, using a measure of recent verbal memory with improved internal behavioral controls so that the same function, naming of different objects, is used as the input and output from memory and the distracting task when the memory must be stored, and second, determination of the effect of serial retrieval of the same item on the increased neuronal activity at initial retrieval. The findings reported provide further insight into the neuronal substrate of human recent verbal memory.

A preliminary presentation of some of these data has appeared in abstract form (Haglund et al., 1990).

\section{Materials and Methods}

Subjects. The subjects included 11 adults on antiepileptic medications undergoing craniotomies for intractable epilepsy under local anesthesia (0.5\% Lidocaine and $0.25 \%$ Bupivacaine). All patients were left hemisphere dominant for language as determined by preoperative intracarotid amytal perfusion (Wada and Rasmussen, 1960). The project and consent form were reviewed and approved by the University of Washington Human Subjects Review Committee. All patients underwent separate informed consent for the surgical procedure and the experimental testing.

Microelectrode recording. The cortical sites for the microelectrode recordings were all within the boundaries of the planned cortical excision. The sites were in the superior and middle temporal gyri, in the first $55 \mathrm{~mm}$ from the tip of the temporal lobe. The local anesthetic and $1 \mathrm{cc}$ of Innovar (fentanyl and droperidol) were given 2-3 hr prior to the language testing. The Trent Wells hydraulic micromanipulator was 


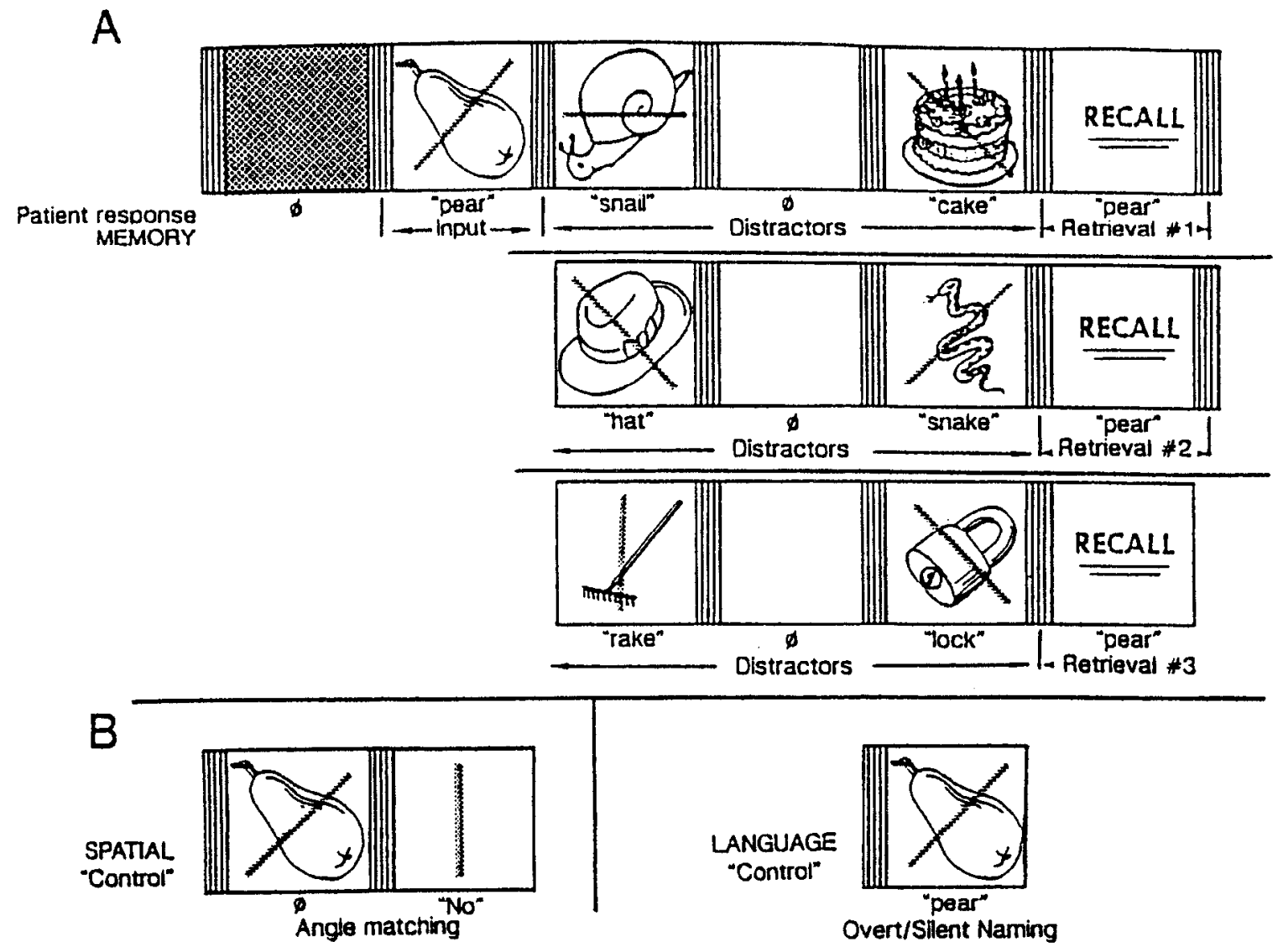

Figure 1. Single trials of the behavioral measures used in this study. A, Memory measure. Each trial consists of the 14 illustrated slides. Below are indicated patient responses and the phase of memory represented by each slide. A subsequent trial of this measure, using different object pictures immediately follows. $B$. "Control" measures: left, one trial of angle matching; right, one trial of naming. Naming was presented twice, once with the instruction to name overtly, the other with the instruction to name silently. Note that physically identical slides are used for memory input, the first slide of the angle matching pair and naming. Identical overt responses are elicited for memory input and overt naming (and no responses with silent naming and the first slide of the angle matching pair).

mounted on the skull clamp post with a small footplate to stabilize the cortex. The footplate did not blanch the underlying blood vessels.

The microelectrodes were primarily stereotrodes that were epoxylitecoated tungsten electrodes electrolytically sharpened to diameters of 1 $5 \mu \mathrm{m}$ (McNaughton et al., 1983; Cawthon et al., 1989). Typical input impedance of the microelectrodes was 3-7 M . Microelectrodes were advanced by the micromanipulator to depths of $1-3 \mathrm{~mm}$. Recordings were checked for lack of injury discharge or epileptiform burst activity but were otherwise unselected. Once stable recordings were obtained the behavioral tasks were begun. Recordings where activity was lost before all behavior tasks were completed were not included in this analysis.

Behavioral tasks. The behavioral tasks were presented as slides, each shown for $4 \mathrm{sec}$. The memory measures consisted of trials each containing 14 slides (Fig. 1A). Each trial began with a blue blank slide, following which the patient viewed an object picture to be named (memory input, MI). Three intervening distraction slides were then viewed. These consisted of two objects to be named and an intervening blank slide. These distractor slides all differed from the object to be retained in memory. In no case did the objects to be named have the word spelled out below the object. Thus, the task involved only object naming. The distractor slides were followed by a slide with the word "recall." This slide was a cue for the patient to recall aloud (R1) the object name that had been pictured on the slide named after the blue slide, and retained in memory for the $12 \mathrm{sec}$ occupied with distractor slides. The serial retrievals were obtained by continuing in the same trial with three more distractor slides (two with different objects to be named and an intervening blank slide) followed by another slide with "recall" where the initial object-naming slide was to be recalled (R2). This sequence was then repeated a third time (with different objects as distractors) so that a total of three serial retrievals were obtained for each trial ( $R 1$, $\mathrm{R} 2, \mathrm{R} 3$ ). In each individual trial, the specific object to be retained in memory was not one of the distractor slides. The patients were briefly trained on the paradigm the evening prior to surgery. Because of time constraints in the operating room, only four to eight trials of this measure were obtained for each patient.

Prior to or following the memory task, the patients performed language tasks where the same slides used for memory inputs and distractors were named overtly and silently in a sequence of 6-12 slides (Fig. $1 B$ ). The patient also performed a spatial matching task using the same object slides (in all tasks these slides had a red stripe across the object) where a red stripe over the object was matched to a following slide with only a red stripe at the same or different angles. The patient stated "yes" or "no" whether the stripes matched or not. Angle matching is a function that has been related to the nondominant hemisphere hased on the effects of lesions (Benton et al., 1975), and likely requires a similar level of attention as does the memory input part of the memory measure. The language tasks were always done just before the spatial matching task. However, all these tasks were done after the memory measure for 14 of the neuronal populations, before the memory measure for two neuronal populations, and between trials of the memory measure for four neuronal populations. The sequence of naming and spatial matching tasks to the memory measure had no apparent effect on the classification of a neuronal population as related to memory, or changes with serial retrieval

Neuronal population analysis. Neuronal activity from the microelectrodes, slide markers, and verbal responses were stored for later analysis on FM tape (frequency response, $100 \mathrm{~Hz}$ to $6.5 \mathrm{kHz}$ ). Using a Macintosh 
Table 1. Changes in neuronal activity on the three major comparisons of this study

\begin{tabular}{|c|c|c|c|c|c|c|c|c|c|c|}
\hline \multirow[b]{2}{*}{ Type } & \multirow[b]{2}{*}{ Pt./unit } & \multirow{2}{*}{$\begin{array}{l}\text { Comparison 1: } \\
\text { Mem./nonmem. }\end{array}$} & \multicolumn{2}{|c|}{ Comparison 2} & \multirow{2}{*}{ Comparison 3: } & \multirow{2}{*}{ Location } & \multirow{2}{*}{$\begin{array}{l}\text { Distal } \\
\text { pole } \\
(\mathrm{mm})\end{array}$} & \multirow{2}{*}{$\begin{array}{l}\text { Depth } \\
(\mathrm{mm})\end{array}$} & \multirow{2}{*}{$\begin{array}{l}\text { Other } \\
\text { behavior }\end{array}$} & \multirow{2}{*}{$\begin{array}{l}\text { Number } \\
\text { of } \\
\text { trials }\end{array}$} \\
\hline & & & Mem. input & $\mathrm{R} 1$ & & & & & & \\
\hline \multirow[t]{4}{*}{ Type 1} & $9123 / 2$ & $\mathrm{mi}+2 ; \mathrm{nL}-2,+4$ & $\mathrm{mi}+1,2,4$ & $\mathrm{R} 1+1$ & fade & STG & 55 & 2 & 0 & 6 \\
\hline & $8931 / 1$ & $\mathrm{mi}+2$ & $\mathrm{mi}+1,2,3$ & $\mathrm{R} 1+1$ & fade & MTG & 40 & 2 & $o N-1$ & 4 \\
\hline & $9117 / 1$ & $\mathrm{mi}-1,3$ & $\mathrm{mi}+1,2$ & $\mathrm{R} 1+1$ & fade & MTG & 45 & 3 & 0 & 8 \\
\hline & $8869 / 2$ & $\mathrm{mi}+2$ & $\mathrm{mi}+1,2$ & $\mathrm{R} 1+1$ & fade & MTG & 40 & 2 & 0 & 6 \\
\hline \multirow[t]{2}{*}{ Type 2} & $9117 / 2$ & $\mathrm{mi}+2 ; \mathrm{oN}-1$ & $\mathrm{mi}+1,2$ & 0 & fade & MTG & 45 & 3 & 0 & 8 \\
\hline & $9006 / 1$ & $\mathrm{mi}+1,2,3 ; \mathrm{oN}-1 ; \mathrm{nL}-4$ & $\mathrm{mi}+1,2,4$ & 0 & fade & MTG & 30 & 3 & N/A & 6 \\
\hline \multirow[t]{4}{*}{ Type 3} & $9032 / 1$ & $\mathrm{mi}+1,4$ & $\mathrm{mi}+1,2$ & $\mathrm{R} 1-3,4$ & 0 & MTG & 45 & 1 & 0 & 5 \\
\hline & $9102 / 1$ & $\mathrm{mi}+1 ; \mathrm{oN}-1$ & $\mathrm{mi}+1$ & 0 & 0 & MTG & 53 & 1.5 & N/A & 8 \\
\hline & $8931 / 2$ & $\mathrm{oN}-2 ; \mathrm{nL}-1,2$ & $\mathrm{mi}+1$ & 0 & 0 & MTG & 40 & 2 & $\mathrm{RL}+2$ & 4 \\
\hline & $8904 / 2$ & $\mathrm{mi}+1,2,3 ; \mathrm{oN}-1,3$ & $\mathrm{mi}+1$ & 0 & 0 & MTG & 35 & 1.5 & $\mathrm{RL}+2$ & 6 \\
\hline \multirow[t]{3}{*}{ Type 4} & $8959 / 1$ & $\mathrm{oN}-1 ; \mathrm{nL}-1$ & 0 & $\mathrm{R} 1+1,2$ & fade & STG & 35 & 1.5 & 0 & 6 \\
\hline & $8904 / 1$ & $\mathrm{mi}+1$ & 0 & $\mathrm{R} 1+1$ & fadc & MTG & 35 & 1.5 & 0 & 6 \\
\hline & $8869 / 1$ & $\mathrm{mi}-1,2$ & 0 & $\mathrm{R} 1+1,3$ & fade & MTG & 40 & 2 & 0 & 6 \\
\hline \multirow[t]{2}{*}{ Type 5} & $9123 / 1$ & $\mathrm{oN}+1,2$ & 0 & 0 & 0 & STG & 55 & 2 & 0 & 6 \\
\hline & $9006 / 3$ & $\mathrm{oN}+1$ & 0 & 0 & 0 & MTG & 30 & 3 & N/A & 6 \\
\hline \multirow[t]{5}{*}{ Type 6} & $8942 / 1$ & 0 & 0 & 0 & fade & MTG & 30 & 3 & $\mathrm{RL}+2$ & 4 \\
\hline & $8942 / 2$ & 0 & 0 & 0 & 0 & MTG & 30 & 3 & $s R-1$ & 4 \\
\hline & $9006 / 2$ & 0 & $m i+3,4$ & $\mathrm{R} 1-4$ & 0 & MTG & 30 & 3 & $\mathrm{~N} / \mathrm{A}$ & 6 \\
\hline & $8947 / 1$ & 0 & $\mathrm{mi}-2,4$ & 0 & 0 & STG & 23 & 1 & N/A & 4 \\
\hline & $9123 / 3$ & 0 & 0 & $\mathrm{Rl}+2$ (speech) & 0 & STG & 55 & 2 & 0 & 6 \\
\hline
\end{tabular}

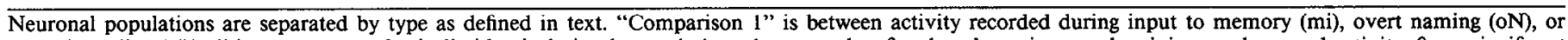

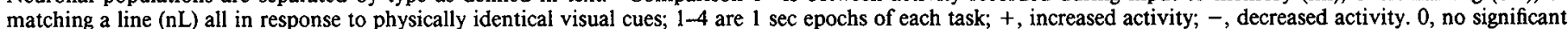

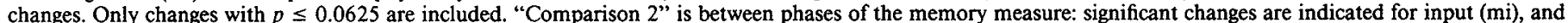

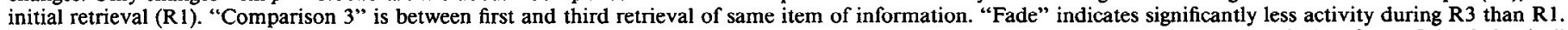

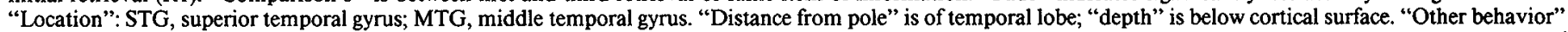

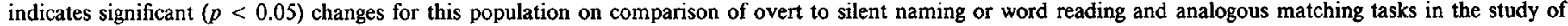
Schwartz et al. (1993). N/A, complete data for these comparisons not obtaincd.

IIx, the analog signals from the FM magnetic tape were digitized at $5000 \mathrm{~Hz}$ per channel. Neuronal populations were identified by amplitude discrimination and the negative and positive deflections of each wave. Amplitude-frequency histograms were constructed to separate recordings into populations based on amplitude.

Recordings obtained during each slide presentation were divided into four equal epochs of $1.0 \mathrm{sec}$. The second epoch $(1.0-1.99 \mathrm{sec})$ always contained the patient's response during the overt naming of objects (memory input, overt naming, recalls, etc.), and the same epochs were then used for the silent and line-matching tasks. The first epoch included perception of the visual stimulus and internal processing; the second epoch, later or delayed internal processing and any overt output, and the third and fourth epochs, the pause between tasks. The following five comparisons were made for each neuronal population.

(1) Changes in neuronal activity during physically identical slides. Physically identical slides were presented as either an input to memory (MI), an object to be named but not retained in memory, or the first slide of a spatial matching pair. Significant changes in activity during memory input on this comparison (or significant decrease in both naming and spatial tasks) would relate a population to recent verbal memory.

(2) Changes in neuronal activity during the memory measure. Averaged across all trials, the same verbal output-enunciating an object name-occurs with memory input, two of the three distracting slides and the initial retrieval (R1), and physically identical slides have been presented for memory input and two of the three distracting slides. Thus, significant changes on this comparison that do not follow these patterns cannot be related to perception or motor output, providing additional evidence of the specificity of changes to recent memory and identifying where in recent memory-MI, storage, or RI-those changes occur.

(3) Changes in neuronal activity during serial retrievals of the same items. As the cue to retrieval for R1, R2, and R3 and the verbal output are the same, changes in neuronal activity indicate an effect of serial retrieval after an additional distraction period.

(4) Changes in neuronal activity during the distractor-naming slide immediately before $R 1, R 2$, and $R 3$. Comparison between these three slides provides controls for the serial retrieval comparisons for several nonspecific effects, such as a general habituation or overall decline in activity.

(5) Changes in neuronal activity during the blank slides before $R I$, $R 2$, and $R 3$. Comparison between these three slides provides another control for nonspecific effects, such as a general habituation or overall decline in activity. In the 20 neuronal populations, there was no evidence of any general habituation during individual trials.

Statistical analyses. Neuronal activity for all epochs of each slide in each of the five comparisons described above was ranked for each trial, and changes from the mean rank for each epoch evaluated with the Wilcoxon signed rank test. Because of the limited time in the operating room, in some cases it was only possible to complcte four trials of the rather long memory measure in the allotted time. If all four trials changed in one direction, either increase or decrease, the Wilcoxon test for an epoch of the comparisons including that test has a $p$ value of 0.062 , slightly above the standard 0.05 (Marascuilo and McSweeney, 1977). Of the 20 neuronal populations analyzed, five included four trials of the memory measure, one included five trials, 11 included six trials, and three had eight trials. For those neuronal populations with only four trials, statements at the usual level of statistical significance for an epoch in a specific population were not possible.

However, a very conservative statistical statement on changes in a specific epoch of a particular comparison for all populations considered together can be made by using the binomial expansion for 8,12 , or 20 epochs, at $p=0.062$ for 20 populations, with significance set at 0.05 , after a Bonferroni correction for the multiple tests in cach comparison. For example, an epoch of the first comparison described above (the physically identical slides used as memory input, overt naming or first slide of the spatial matching task) would show an overall significant change, if four or more of the 20 populations showed an increase at $p$ $\leq 0.062$, since the binomial expansion at this $p$ indicates that the chance occurrence of four or more events in the same direction in one of the 12 epochs of this comparison is 0.004 , which is below the 0.05 
Figure 2. A, Proportion of all 20 neuronal populations with changes in activity at $p \leq 0.062$ for each $1 \mathrm{sec}$ epoch of comparison 2, between input, distractor, and R1 phases of the memory measure. Horizontal broken line indicates number of populations (5) that might be expected by chance (based on binomial expansion for 20 tests at $p$ 0.05 with Bonferroni correction). More populations demonstrated increased activity with epochs 1 and 2 of memory input and epoch 1 of initial retrieval than expected by chance. $B$, Same as for $A$, but for comparison 3, between the three serial retrievals. Horizontal broken line is number of populations (4) that might be expected by chance for this many tests. More populations than expected by chance with increased activity in epochs 1 and 2 of the initial retrieval are again evident, and this increased activity disappeared with serial retrievals, so by epoch 3 of the third retrieval there are significantly more populations with decreased activity.
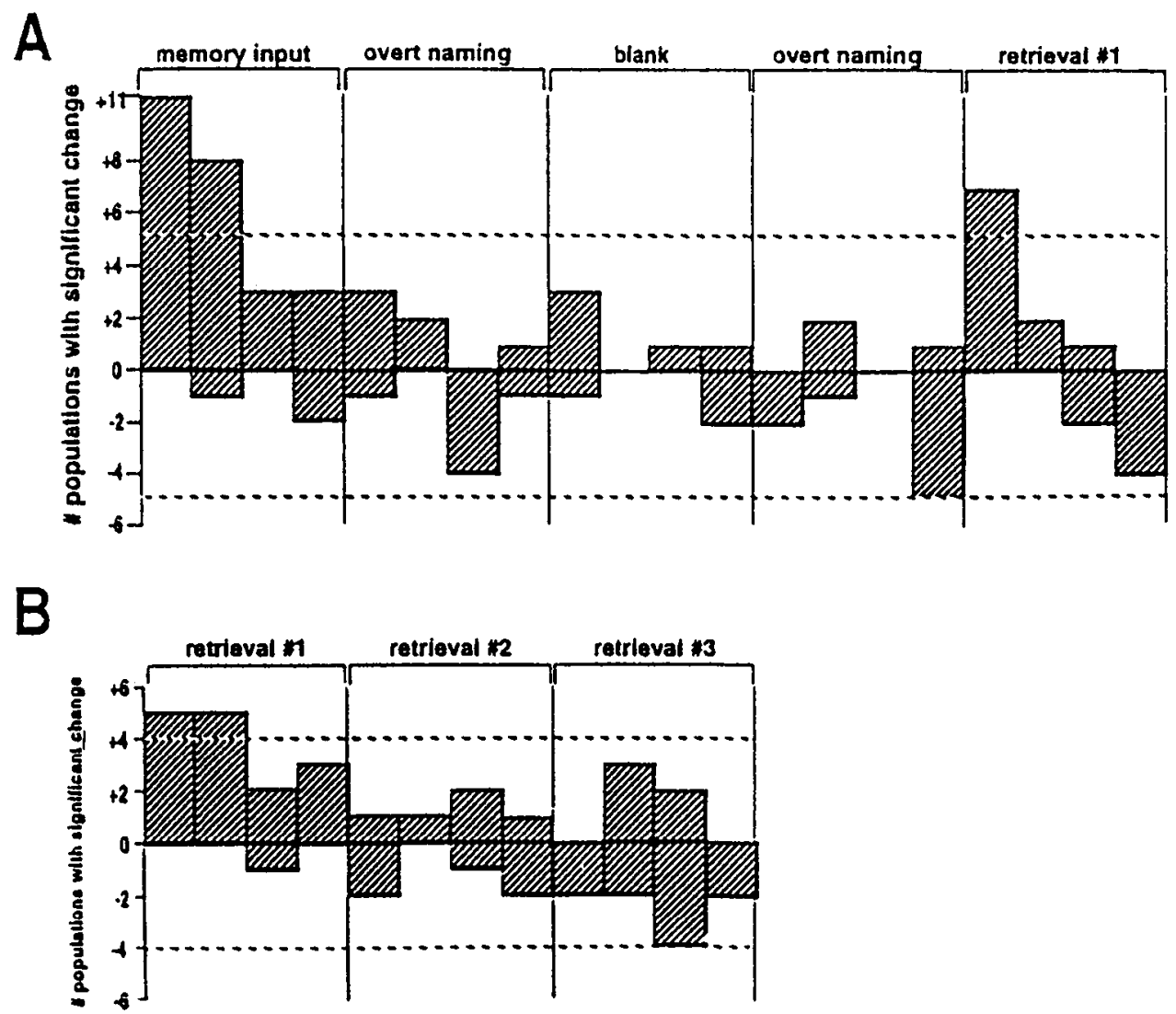

level after the Bonferroni correction for the 12 tests. This method allows conventional statistical statements of significance to be made concerning the population of neurons, but not the individual neuronal recordings.

\section{Results}

Neuronal populations

Twenty stable neuronal populations, free of injury discharge, bursting activity, pulsation artifact, or electrode movement, were recorded from 11 patients. All recordings were made in lateral temporal neocortex (superior and middle temporal gyri). All recordings were made in areas that had shown little or no evidence of epileptiform activity during 30-45 min of electrocorticography. The majority of the recordings were made in the middle temporal gyrus $(n=15)$ with the remainder in the superior temporal gyrus $(n=5)$. Recording depths varied between 1 and $3 \mathrm{~mm}$ below the pial surface. Using the procedures already described, single populations were identified at four recording sites; two populations at five recording sites; and three populations at two sites. At those recording sites where more than one population was identified, the neuronal populations always showed differences in their firing frequency, amplitude, and behavioral responses. The average baseline firing rate was typically low for these lateral temporal cortex neurons $(<1 \mathrm{~Hz})$; however, some neurons showed increases in activity up to $30 \mathrm{~Hz}$ during specific behavioral mcasurcs.

\section{Comparison 1: relation to memory input, language, or spatial matching}

Of the 20 neuronal populations, five (20\%) showed no change related to any behavior on this comparison. Two (10\%) showed changes only to language tasks (overt naming or silent naming).
The 13 remaining neuronal populations (65\%) showed significant increases with memory input compared to the language and spatial matching tasks (Table 1).

\section{Comparison 2: relation between memory input, storage, and $R I$}

A significant proportion of all 20 neuronal populations show changes during initial epochs of memory input (MI) and/or the initial retrieval, and not during the distraction period (Fig. $2 A$ ). When the 13 neuronal populations related to memory by comparison 1 are considered alone, $10(77 \%)$ had increases during the first $1 \mathrm{sec}$ epoch of memory input. The probability of this many or more populations showing increases during the first epoch of the memory input is $p<0.0001$ (binomial expansion for $p=0.062$ and $n=13$ ). Sustained increases, defined as increases in neuronal activity beyond the first epoch, were found in $60 \%$ ( 6 of 10 ) of these neurons ( $p<0.0001)$. Sustained increases in the neuronal activity lasting into the third and fourth epochs occurred in three populations $(30 \%)$. In these populations, increased activity continues beyond the epoch related to overt responses.

Initial retrieval (Rl) evoked significant changes in the neuronal activity in seven (53\%) of these 13 neuronal populations. Increases were predominantly in the first epoch of R1 when the subject was preparing to retrieve the memory input $(p<0.001)$. The significant changes in both $\mathrm{MI}$ and $\mathrm{R} 1$ were predominantly in the first two epochs, indicating a relationship to the task. These findings for the memory input and $\mathrm{R} 1$ contrast with activity during the distraction where the only significant change $(p<0.05)$ was a decrease in activity in the last epoch of the second overt-naming distractor slide. When changes in activity 
of an epoch of individual populations that differ from surrounding epochs at $p \leq 0.0625$ are considered, four populations demonstrated increased activity in both $\mathrm{MI}$ and $\mathrm{R} 1$ (31\%), six populations only MI (46\%), and three only R1 (23\%).

This comparison within the memory task is potentially independent of comparison 1 , that established a population as related to memory. Indeed, three of the seven neuronal populations not related to memory on that initial comparison showed some changes at the $p \leq 0.0625$ level on the comparison within the memory task. However, two of the changes were in a pattern rarely seen with memory neurons: one with decreased activity at memory input, the other with only very late changes (epoch 3 or 4 ). The remaining "nonmemory" population showed increased activity in the early epochs of initial retrieval, probably representing another population with only $\mathrm{R} 1$ changes.

\section{Comparison 3: changes in neuronal activity during serial retrievals}

Changes in activity during serial retrievals were then determined for all neuronal populations (Fig. $2 B$ ) and those related to memory in comparison 1 . The majority of neuronal populations related to memory $(69 \%)$ decreased activity from the first to the third retrieval. In $\mathrm{R} 1$, the majority of changes (15 of 16) are in the positive direction while in $\mathrm{R} 2$ the percentage of positive changes decreases (5 of 10), decreasing further in R3 (5 of 16). This fading of neuronal activity is a progressive process as indicated by the comparisons of R1 to R2 and R2 to R3 (Fig. 3). The probability of finding seven increases in the first epoch of $\mathrm{R} 1$ compared to six decreases of $\mathrm{R} 2$ is very low (both $p<$ $0.00001)$. Similarly, the findings of five increases in the second epoch of R2 $(p<0.0001)$ and five decreases in the third epoch of R3 $(p<0.001)$ also point toward a progressive fading of the neuronal activity between later retrievals. The early increases appear to be diminished first, since in the R2-R 3 comparison the fading occurs during the later epochs.

Fading with serial retrieval was a characteristic of all seven memory populations with increased activity at initial retrieval, including all populations with increased activity on both memory input and the initial retrieval. The $\mathrm{R} 1, \mathrm{R} 2, \mathrm{R} 3$ comparison is potentially independent of other measures, so changes could be present in populations showing relations to memory input, or even those not related to memory. Two (of six) populations with only increased activity at memory input showed fading, as did one of the seven "nonmemory" populations.

\section{Comparisons 4 and 5: changes in neuronal activity during the} distractor-naming and blank slides immediately before $R 1$, $R 2$, and $R 3$

The fading of neuronal activity between R1-R2-R3 could represent a general trend for the neuronal activity to decrease during an individual trial. If this hypothesis is correct, there should be a general decrease in activity in the overt-naming slide immediately preceding the $\mathrm{R} 1, \mathrm{R} 2$, and $\mathrm{R} 3$ slides. In Figure 4 , this comparison of the number of populations with changes at $p \leq$ 0.062 in the three overt-naming distractor slides shows no significant epochs. As previously mentioned, the comparison of the blank slides between MI-R1, R1-R2, and R2-R3 also showed no significant changes in neuronal activity during any of the epochs. These findings show that there is not a general trend for neuronal activity to fade during the internal controls; rather, the fading of neuronal activity is specific to the serial retrievals.

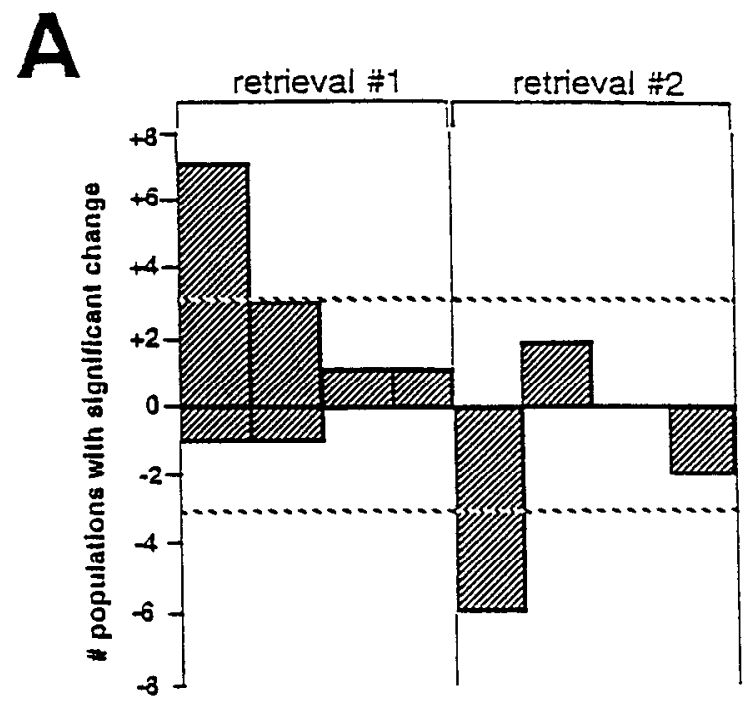

5

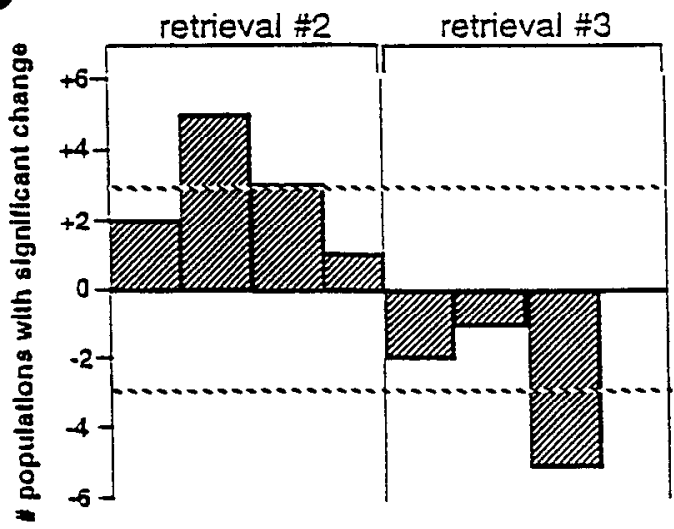

Figure 3. Changes in activity between first and second retrievals from memory $(A)$ and second and third retrievals $(B)$ for the 13 neuronal populations related to memory by comparison 1. Broken line is number of populations expected to show changes on a chance basis at $p \leq 0.05$. Significantly more populations than expected by chance have increased activity in epoch 1 of initial retrieval compared to decreased activity in epoch 1 of the second retrieval, while when second is compared to third retrieval, a significant number of populations with increased activity are identified in second and third epoch of the second retrieval compared to decreased activity in the third epoch of the third retrieval. These findings indicate a progressive fading of increased activity with serial retrieval of the same information.

\section{Changes in neuronal activity related to the familiarity of objects}

Another possible explanation for the fading of neuronal activity during the serial retrievals involves habituation because of the familiarity of objects being presented. Some of the object slides that were used as input to memory were also used as distractor slides on other trials. Mcmory input slides that demonstrated fading of neuronal activity during serial retrievals, when used in separate trials as distractors, were separated by $6-17$ slides and repeated one to four times. A significant increase in the first epoch of the initial presentation of an object compared to the first epoch of the last presentation would suggest that decreased neuronal activity during serial retrievals is due to familiarity of the object being named. No significant changes were seen. There is also no difference when those neuronal populations with fad- 


\section{A}

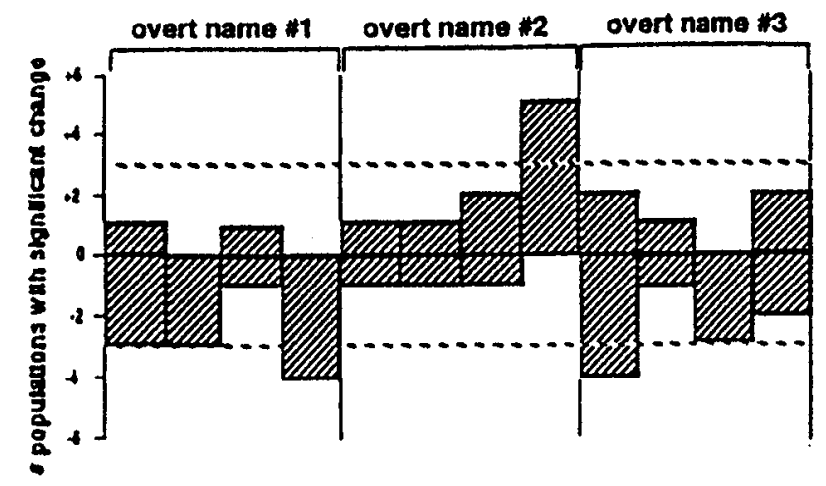

Figure 4. Control for declining activity as an explanation for effects illustrated in Figures $2 B$ and 3. Activity in the naming slide preceding each retrieval is compared for all three retrievals $(A)$, for first compared to second retrieval $(B)$, and for second compared to third retrieval $(C)$. No significant changes are present.
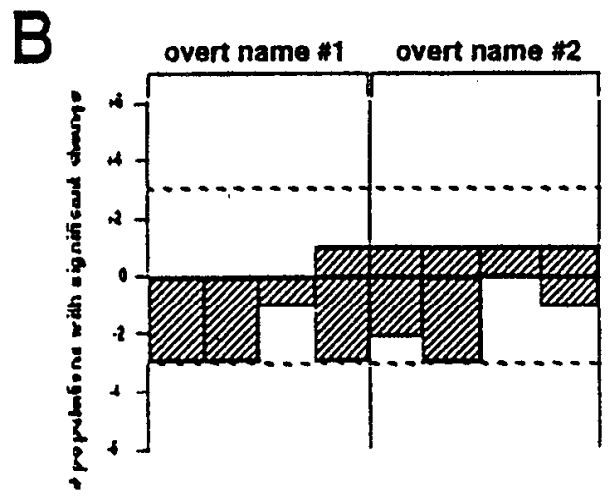
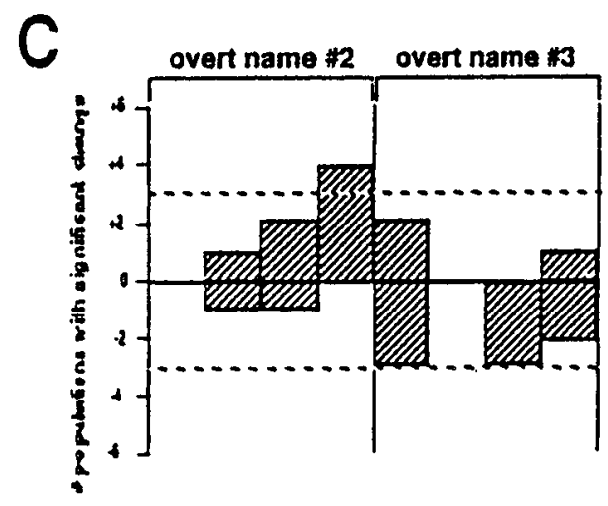

ing on serial retrieval (Fig. $5 A$ ) are compared to those that did not fade (Fig. $5 B$ ). Indeed, the first presentation actually has more significant epochs in the negative direction than in the positive direction. These negative findings, in addition to the negative finding for a general trend toward decreasing neuronal activity in individual trials, emphasize that the decreases in activity during serial retrievals are related to memory.

\section{Location of neuronal populations associated with memory}

For the purposes of anatomical localization, the 13 memoryrelated neuronal populations were divided into (1) four populations with increases (at the $p \leq 0.062$ level) during MI and R1 with fading during the serial retrievals (type I), (2) two populations with increases during MI and fading during serial retrievals (type II), (3) four populations with increases during MI without RI changes and no fading (type III), and (4) three populations with no change during MI but increases with R1 and fading during serial retrievals (type IV). The nonmemory populations were divided into language-related $(n=2$; type $\mathrm{V})$ and five populations with no change during any behavioral measure (type VI) (Table 1).

The anatomical location of the neuronal recording sites were recorded for the gyrus (middle or superior temporal); the distance from the temporal tip (range, 23-55 $\mathrm{mm}$ ); and the depth below the pial surface (1-3 mm). No major pattern in the anatomical location and type of neuronal population was obvious, except that the memory-related neuronal populations in the middle temporal gyrus were posterior compared to the more anterior nonmemory neuronal populations (Fig. 6A). The superior temporal gyrus contained no pattern in the anatomical location or in the type of neuronal population.
In the comparison of the microelectrode depth and its relationship to the type of memory-related neuronal populations, one significant theme was observed (Fig. $6 B$ ). There were no differences between the depth when comparing neurons with changes during memory input, initial retrieval, or fading during serial retrieval. However, when comparing those memory neurons with increases in MI and fading of R1-R3 (neuronal types I, II) to those memory neurons without this combination (neuronal types III, IV), a significant difference was observed. Those neurons with increases during MI and fading during serial retrievals $(n=7$, types I, II) were deeper $2.5 \pm 0.2 \mathrm{~mm}$ compared to those memory-related neurons ( $n=6$, types III, IV) without both these changes, $1.6 \pm 0.1 \mathrm{~mm}(p=0.028)$.

\section{Memory-related neurons and language}

Comparison 1 indicated a separation between neurons related to memory and those rclatcd to naming. In addition, as part of another study (Schwartz et al., 1992), many of these same neurons were analyzed for changes during additional behavioral measures comparing overt to silent word reading, and a wordreading spatial matching task, as well as comparing overt to silent naming and the analogous spatial matching task. Of the memory-related neuronal populations $(n=13), 11$ populations were analyzed for these additional behavioral measures. Only three of the 11 neuronal populations $(27 \%)$ related to memory show any change during these other behavioral measures. In the three neuronal populations that did show changes with the other measures, two were increases in activity during the spatial task in response to a word with a line across it (rather than an object and line) and one was a decrease in activity during the first epoch of the overt naming task. These comparisons provide 

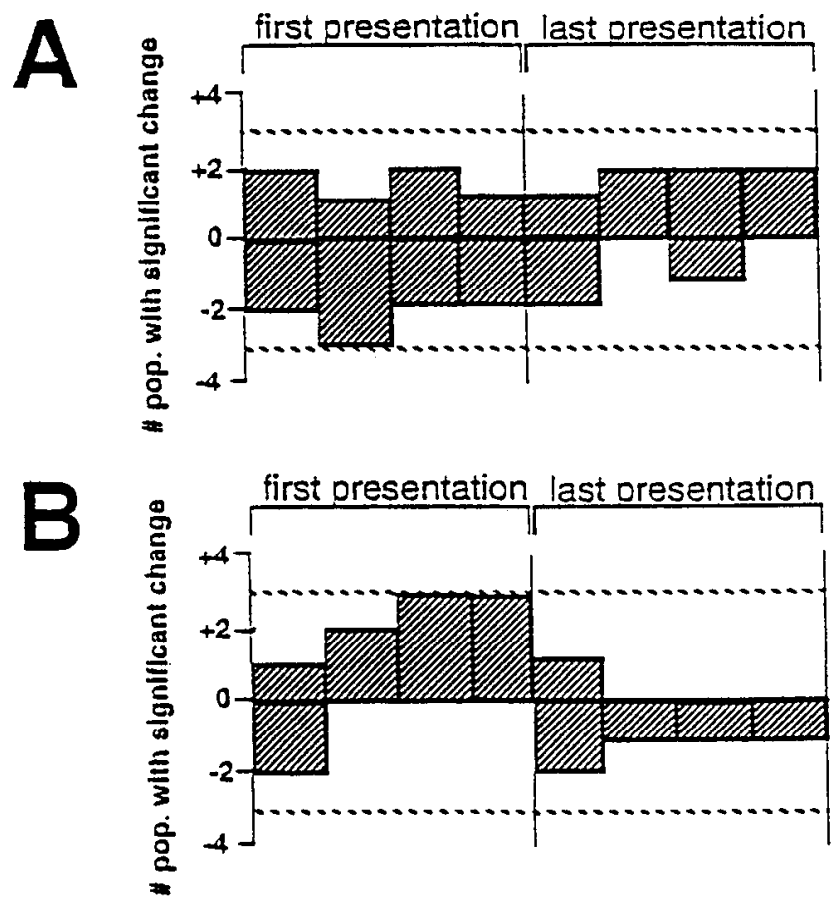

Figure 5. Control for general habituation as an explanation for effects illustrated in Figures $2 B$ and 3. Activity is compared during an initial and subsequent naming of the same object picture for populations showing fading of activity with serial retrieval $(A)$ and for populations without fading $(B)$. There is no evidence of reduced activity with repeated naming of the same object picture.

further evidence that the memory-related neuronal populations are primarily related to memory and not other language or spatial behaviors.

Of the seven neuronal populations not related to memory, only four were analyzed for the other behavioral measures. However, three of the four $(75 \%)$ had changes related to the other behavioral measures. One unit related to language in the comparison of this study had increases in activity in the first epochs of the overt and silent reading tasks. Two units with no behavioral changes on comparison 1 of this study showed reciprocal changes to silent reading and the spatial task in response to a reading stimulus. Although these comparisons have a small sample size, a minority of memory-related neuronal populations and the majority of nonmemory neuronal populations show changes with other behavioral measures such as naming and reading.

Seven patients had microelectrode recordings separable into two or more neuronal populations (Table 1). Three patients, including both patients with three populations, show very different behavioral correlates in the nearby populations. The other four patients show changes with the memory measure for both populations, but no two nearby populations had significant changes in the same portion of the memory measure.

\section{Discussion}

Changes in neuronal activity during memory input and initial retrieval

This study confirms the finding in our earlier study that a large proportion of neurons sampled from left dominant anterior temporal neocortex increase activity with recent verbal memory
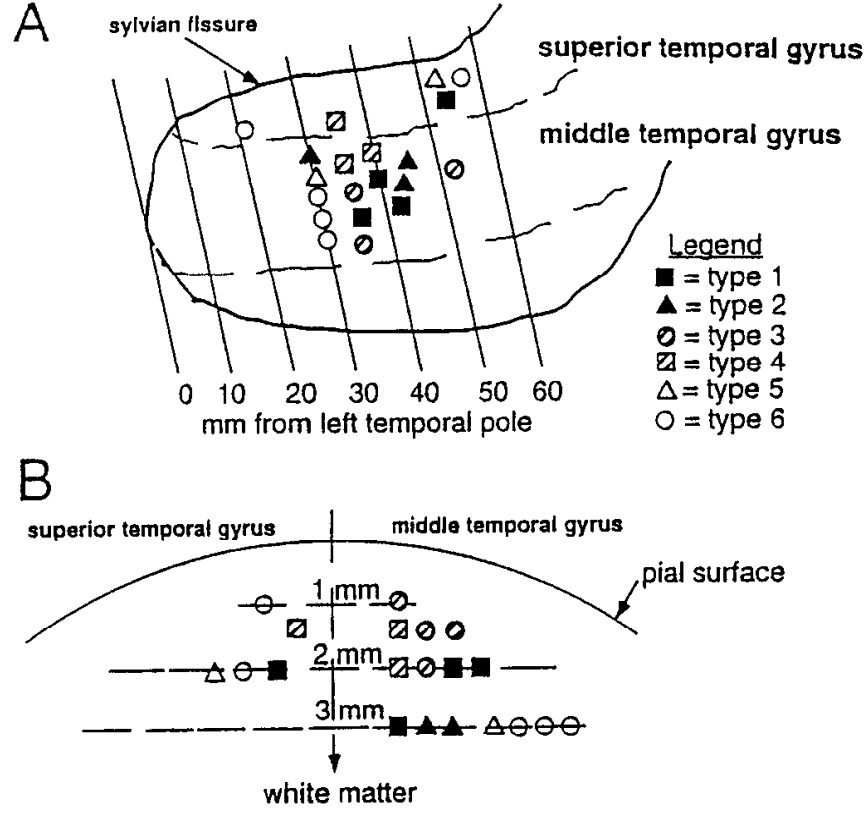

Figure 6. Location of types of changes in neuronal activity with memory measure. $A$, Lateral projection. $B$, Cortical depth. Types of changes in activity are defined in Results.

measures. In the present study we have used two comparisons to relate a population to memory: (1) changes on the memory measure when the visual cues were physically identical for three different tasks-memory input, a language task without any memory component, and a spatial matching task-as well as having the same verbal output for the memory and language tasks; and (2) changes within the recent verbal memory measure based on the input-distractor-output paradigm of Peterson and Peterson (1959). In the first comparison, $13(65 \%)$ of the 20 sampled populations were rclatcd to memory input by both measures. Our previous study showed a similar proportion of populations related to memory, 12 of $17(70 \%)$ (Ojemann et al., 1988). The probability of seeing a relation to memory on both measures for an individual population is quite small and suggests that a large proportion of neurons in the lateral temporal cortex are dedicated to processing memory input and the initial retrieval from memory.

The second comparison adds another dimension to the understanding of the role of the lateral temporal lobe in memory. Increases in neuronal activity in the second comparison were related to memory input in $77 \%$ of the memory-related populations as defined by the first comparison, and $58 \%$ in the previous study (Ojemann et al., 1988). This activity occurs essentially every time new information is encountered, for only that patterr would achieve the significance levels required to relate a ncuronal population to the input memory phase in either study. This increased activity when a new item enters recent verbal memory is often relatively long lasting, extending beyond the time required to identify the item verbally in $30 \%$ of the neuronal populations with memory input changes in the present study and $50 \%$ in the earlier study. Increased activity at initial retrieval was evident in $53 \%$ of the memory populations of the present study and $58 \%$ of the earlier study.

These findings are supported by microelectrode recordings from monkey inferior temporal lobe during a visual delayed 
match-to-sample task. Fuster and Jervey (1982) found a large proportion of neurons with changes in neuronal activity during the sample and match after a 15-20 sec delay (sample: changes, $96 \%$; no change, $4 \%$ ). In contrast, a much smaller percentage of neurons showed changes in the intervening delay between the sample and match (changes, 44\%, no change, 56\%). This type of change was less evident when the delay was filled with distractors (Fuster, 1990). These findings demonstrate that a large proportion of neurons in the primate inferior temporal region also show changes with visual memory measures.

\section{Changes in neuronal activity during serial retrievals}

The present study indicates that the increase in neuronal activity at the initial retrieval is not sustained through subsequent retrievals of the same item. A reduction is already apparent by the second time an item is retrieved and even more so by the third. The reduction in activity is quite specific to items in recent explicit verbal memory. No overall reductions in neuronal activity occurred nor did reductions occur with serial naming of the same items when they were not to be retained in memory. Thus, there is no evidence from our data for an overall habituation or for effects of novelty, per se.

This transient increased activity of many temporal neocortical neurons at memory entry is apparently essential for retention of items. The verbal memory deficit after left temporal lobe resections has been related in part to the extent of the neocortical removal (Milner, 1967; Ojemann and Dodrill, 1985). With surface electrical stimulation during memory entry at many sites in left anterior temporal lobe, although the object can be correctly named, it is not retained in recent verbal memory (Ojemann, 1978, 1983). Surface stimulation presumably alters the increased activity at memory entry. The reduction in this activity with serial retrieval occurs in most memory-related temporal neocortical neurons. This suggests that if neuronal activation is part of retaining or retrieving an item that has successfully entered human memory, as is suggested by invertebrate studies of conditioning (Hawkins et al., 1983), those activated neurons are either located elsewhere or are a very small subset of temporal cortical neurons. The widespread initial increase in activity at memory entry (and to a lesser extent at initial retrieval) is perhaps best viewed as a promoter for retention of an item in memory. Damasio has proposed that recall involves simultaneous activation of multiple small cortical "convergence zones" (Damasio, 1990). Perhaps the widespread temporal activity at memory entry sets up the convergence zones for this memory, or the process that "binds" them together. Activation of smaller ncuronal populations then would be required for subsequent retrieval, once the binding phenomenon has taken place after memory input and initial retrieval.

Although the present study was not specifically a test of learning, correct serial retrieval often leads to learning as evidenced by more accurate later retrieval. Thus, learning of an item of information seems to involve this process of reducing the neuronal activity involved in retrieval of that item. Similar evidence has come from both animal and human studies. The activity of primate inferior temporal cortex neurons increased with the sample and/or match in a visual delayed match-to-sample task; however, these changes were not sustained, as further repetitions led to a decrement of the response (Bayliss et al., 1987; Bayliss and Rolls, 1987). Just as the response of temporal lobe neurons to visual memory input and retrieval appear to be widely distributed, the fading of neuronal activity with serial recall of an item entered into memory appears to be distributed across multiple areas of the primate temporal visual cortex. In human studies, patients with lesions in the temporal lobe show marked impairment of recall in paired-association tasks (Milner, 1967, 1971). Positron emission tomographic (PET) studies also show a shrinkage in areas of initially increased blood flow during learning of a motor (Seitz et al., 1990) or a semantic task (Raichle, 1990).

\section{Changes in neuronal activity during other behavioral measures}

The present study, like previous studies of human temporal lobe neuronal activity during measures of higher functions (Haglund et al., 1993; G. Ojemann et al., 1988, 1990; J. Ojemann et al., 1992) found considerable specificity to the behavior eliciting changes in activity of a particular neuronal population. Only 2 of the 13 "memory units" showed changes in activity with any other measured hehavior that was in the same direction as the memory change, in both cases to the spatial matching task involving reading stimuli. These two memory units showed a reciprocal change in activity between memory and overt naming, and one other memory unit showed reciprocal activity between memory input and overt naming. Thus, most neurons are apparently activated by only one of the class of memory, language, or spatial behaviors. This degree of specificity is somewhat surprising, given the large proportion of the sample responding to memory. However, frequent separation between recent verbal memory and language mechanism was also a finding in stimulation mapping studies of temporal cortex (Ojemann and Dodrill, 1985; Ojemann, 1978, 1983).

In our other studies of changes in neuronal activity during language and visuospatial measures (Schwartz et al., 1992; Ojemann et al., 1992), nearby neuronal populations recorded by the same microelectrode usually had different behavioral correlates. In the present study, two-thirds of nearby neuronal recordings responding to memory demonstrated changes to memory in all nearby populations - though in somewhat different ways. No nearby populations altered activity during exactly the same memory phases. Based on the likely consequence of resection, left anterior temporal neocortex is probably more essential for explicit verbal memory than for language or spatial functions. Because of the invasive nature of microelectrode recordings, little is known about the activity in essential language areas. However, the present study suggests that the presence of a high proportion of neurons, including nearby neurons, changing activity with a behavior, may be a feature of cortical areas essential for that behavior.

\section{References}

Bayliss GC, Rolls ET (1987) Responses of neurons in the inferior temporal cortex in short term and serial recognition memory tasks. Exp Brain Res 65:614-622.

Bayliss GC, Rolls ET, Leonard CM (1987) Functional subdivisions of temporal lobe neocortex. J Neurosci 7:330-342.

Benton A, Hannay H, Varney N (1975) Visual perception of line direction in patients with unilateral brain disease. Neurology 25:907910.

Cawthon D, Ojemann G, Lettich E, Kalk D, Percival D (1989) Human extracellular stereotrode analysis of neuronal ensemble dynamics during language tasks. Soc Neurosci Abstr 15:302.

Damasio AR (1990) Synchronous activation in multiple cortical regions: a mechanism for recall. Semin Neurosci 2:287-296.

Fedio P, Van Buren JM (1974) Miemory deficits during electrical stimulation of speech cortex in conscious man. Brain Lang 1:29-42.

Fuster JM (1990) Neuronal discrimination and short-term memory 
in association cortex. In: Neurobiology of higher cognitive function (Scheibel A, Wechsler A, eds), pp 85-101. New York: Guilford.

Fuster JM, Jervey JP (1982) Neuronal firing in the inferotemporal cortex of the monkey in a visual memory task. J Neurosci 2:361375.

Fuster JM, Bauer RH, Jervey JP (1981) Effects of cooling inferotemporal cortex on performance of visual memory tasks. Exp Neurol 71: $398-409$.

Haglund MM, Ojemann G, Cawthon D, Lettich E (1990) Neuronal activity in human temporal lobe with multiple memory retrievals. Soc Neurosci Abstr 16:760.

Haglund MM, Ojemann GA, Lettich E, et al. (1993) Dissociation of cortical and single unit activity in spoken and signed languages. Brain Lang 44:19-27.

Hawkins R, Abrams T, Carew T, Kandel E (1983) A cellular mechanism of classical conditioning in Aplysia: activity dependent amplification of presynaptic facilitation. Science 219:400-405.

Marascuilo L, McSweeney M (1977) Nonparametric and distributionfree methods for the social sciences. Monterey, CA: Brooks/Cole.

McNaughton B, O'Keefe J, Barnes C (1983) The stereotrode: a new technique for simultaneous isolation of several single units in the central nervous system from multiple unit records. J Neurosci Methods 8:391-397.

Milner B (1967) Basic mechanisms suggested by studies of temporal lobes. In: Basic mechanisms underlying speech and language (Millikan C, Darley F, eds), pp 122-133. New York: Grune and Stratton.

Milner B (1971) Disorders of learning and memory after temporal lobe lesions in man. Clin Neurosurg 19:421-446.

Ojemann GA (1978) Organization of short-term verbal memory in language areas of human cortex: evidence from electrical stimulation. Brain Lang 5:331-340.

Ojemann GA (1983) Brain organization for language from the perspective of electrical stimulation mapping. Behav Brain Sci 6:189230 .
Ojemann GA, Dodrill CB (1985) Verbal memory deficits after left temporal lobectomy for epilepsy: mechanism and intraoperative prediction. J Neurosurg 62:101-107.

Ojemann GA, Dodrill CB (1987) Intraoperative techniques for reducing language and memory deficits with left temporal lobectomy. In: Advances in epileptology, Vol 16 (Wolf P, Dam M, Janz D, Dreifuss $F$, eds), pp 327-330. New York: Raven.

Ojemann GA, Creutzfeldt OD, Lettich E, Haglund MM (1988) Neuronal activity in human lateral temporal cortex related to short-term verbal memory, naming and reading. Brain 111:1383-1403.

Ojemann GA, Cawthon DF, Lettich E (1990) Localization of physiological correlates of language and verbal memory in human lateral temporoparietal cortex. In: Neurobiology of higher cognitive function (Scheibel AB, Weschler AF, eds), pp 185-202. New York: Guilford.

Ojemann J, Ojemann G, Lettich E (1992) Neuronal activity related to faces and matching in human right nondominant temporal cortex. Brain 115:1-13.

Petcrson L, Peterson M (1959) Short-term retention of individual verbal items. J Exp Psychol 58:193-198.

Raichle M (1990) Exploring the mind with dynamic imaging. Semin Neurosci 2:307-315.

Schwartz T, Ojemann G, Haglund M, Lettich E (1992) Cerebral lateralization of neuronal activity during naming, reading and line-matching. Epilepsia 33[Suppl 3]:120.

Seitz R, Roland P, Bohm C, Grertz T, Stone-Elander S (1990) Motor learning in man: a positron emission tomographic study. Neuroreport $1: 17-20$.

Squire L (1987) Memory: neural organization and behavior. In: Handbook of physiology, sec 1 , The nervous system, Vol V, Higher functions of the brain, Pt 1 (Mountcastle V, Plum F, Geiger S, eds), pp 295-372. Bethesda, MD: American Physiological Society.

Wada J, Rasmussen T (1960) Intracarotid injections of sodium amytal for the lateralization of cerebral speech dominance. J Neurosurg 17: 266-282. 